\title{
Efficiency Estimation of Infrared Heating Systems for Mounting Electronics Modules
}

\author{
Vladimir L. Lanin ${ }^{1}$, Alexandr I. Lappo ${ }^{2}$ \\ 1 Belarus State University of Informatics and Radioelectronics, Minsk, Belarus, vlanin@bsuir.by \\ 2 Belarus State University of Informatics and Radioelectronics, Minsk, Belarus, lappo@bsuir.by
}

\begin{abstract}
The efficiency of shortly - and middle-wave infrared (IR) sources of heat applied to mounting and demounting of electronic modules is sized up. Thermal fields models analysis shows IR lamps irregularity of heating of the PCB and packages of electronic components. For ceramic IR radiator irregularity of heating of the PCB makes $8-13^{\circ} \mathrm{C}$, the temperature SMD components packages differs from plate temperature on $5-20^{\circ} \mathrm{C}$. Application middle-wave ceramic IR sources allows to attain higher uniformity of heat in a working area and to ensure optimal temperature profile at mounting and demounting of surface-mounted electronic components.
\end{abstract}

Keywords: mInfrared radiation, Heat sources, Mounting, Demounting, Electronic modules.

\section{Introduction}

The use of IR sources allows doing a local heating during the installation of electronic modules, reducing the heating time of the product and reducing the risk of electronic components damage. Heating by IR radiation has a number of technological advantages, but their implementation depends on the correct choice of the heating source and the design of the IR heating system. For a reasonable choice of sources of infrared heating, it is necessary to analyze the thermal fields of heated objects, to estimate the effect of the distance from the heater to the PCB on the uniformity and heating rate $^{[1]}$.

Nowadays the perfect method is the local IR heating ${ }^{[2]}$, which is produced by a focused beam of infrared radiation only in places of soldering. Required procedure in this case is heating the board from below to prevent its deformation. IR ceramic heaters due to the high temperature of heating and high inertia maintain a stable temperature, which is very important for lead-free soldering. In IR soldering stations, radiators are used, and it helps to reach a high temperature as soon as possible ${ }^{[3]}$.

At IR soldering Ball Grid Arrays (BGA) on PCB with some optical setups it is necessary have uniformity thermal distribution at the surface of $\mathrm{BGA}^{[4]}$. In this paper thermal fields of shortly- and middle-wave IR sources are optimized for mounting SMD in electronic modules

\section{MODELING}

For the research was developed a model 4-layer PCB 40x40 mm and components installed on it in BGA, QFP and SMD cases-0805, 1206, 1210 were developed. The distance from the heating elements to the board was $20 \mathrm{~mm}$. The result of modeling is the thermal fields on the surface of the module with surface mounted components ${ }^{[5]}$.

Copyright (C) 2018 MVladimir L. Lanin et al.

doi: $10.18686 /$ esta.v5i1.68

This is an open-access article distributed under the terms of the Creative Commons Attribution Unported License

(http://creativecommons.org/licenses/by-nc/4.0/), which permits unrestricted use, distribution, and reproduction in any medium, provided the original work is properly cited. 
Analysis of the thermal fields (Figure 1a) shows that the uneven heating of the PCB for the halogen IR incandescent lamp $300 \mathrm{~W}$ is $45-55^{\circ} \mathrm{C}$, the main heating is concentrated in the center, where it reaches a temperature peak of $200-205^{\circ} \mathrm{C}$, while the edges do not exceed $140^{\circ} \mathrm{C}$. On the bodies of electronic components, the temperature difference is $90-100^{\circ} \mathrm{C}$. For the ceramic IR heater Elstein SHTS / 4 (Fig. 1b), the uneven heating of the PCB is $8-13^{\circ} \mathrm{C}$, the temperature of the shells of surface mounted components differs from the temperature of the PCB: BGA at $28-32^{\circ} \mathrm{C}$, QFP-24-26 $2{ }^{\circ}$ and SMD-5-20 ${ }^{\circ} \mathrm{C}$.

To optimize the parameters of IR heating, the heating rate and the distribution of thermal fields were modeled as a function of the distance between the heating element and the electronic module. On the temperature-time dependences (Fig. 2), it is seen that as the distance to the electronic module increases, the heating rate decreases twice for every 10 $\mathrm{mm}$ for short-wave heaters (halogen IR lamp) and 1.5 times for medium-wave heaters (ceramic heating element). At a distance of more than $20 \mathrm{~mm}$ IR heating becomes uniform, the temperature spread does not exceed $3-5 \%$ for components and $5-7 \%$ for a $\mathrm{PCB}$

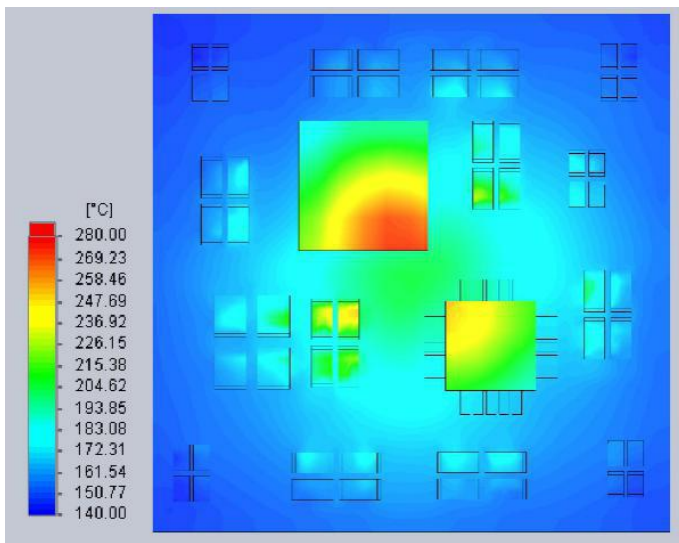

a

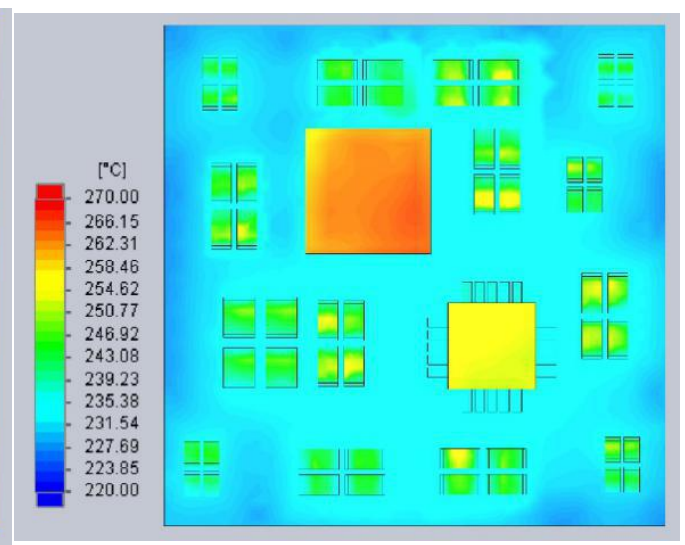

b

Fig. 1. Thermal fields on the surface of the electronic module: a-halogen IR lamp 300W, b -ceramic-IR Heater Elstein SHTS / 4 (simulation).

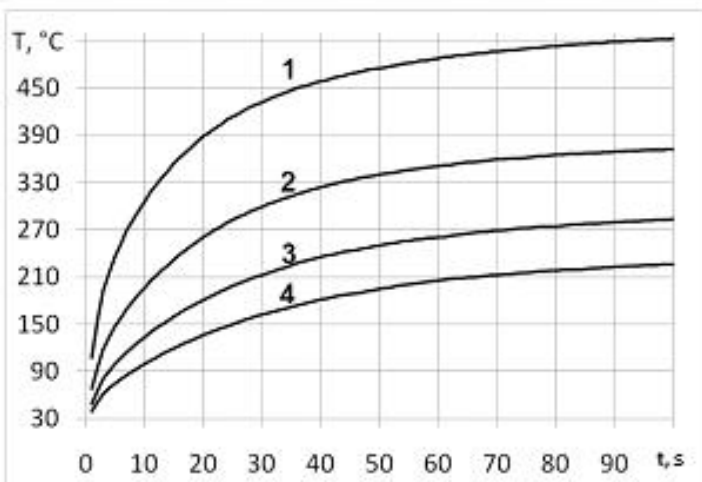

a

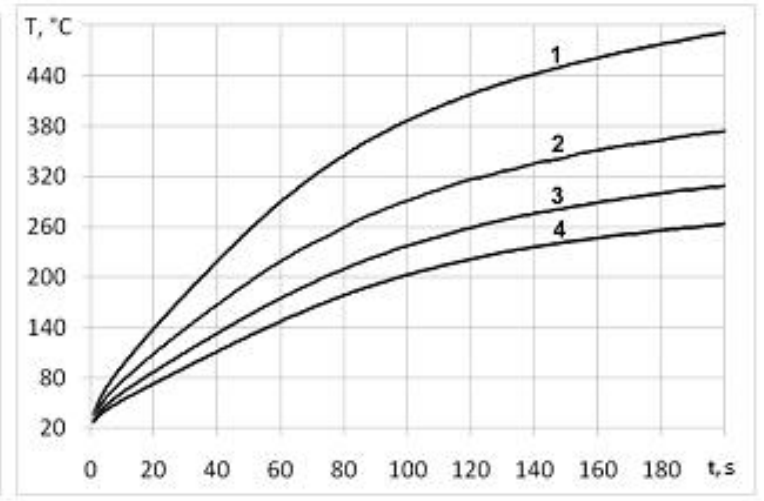

b

Fig. 2: Temperature-time dependencies: a-halogen IR lamp 300W, b - ceramic IR heater Elstein SHTS/4. With distances up to the board: 1-10 mm, 2-20 mm, 3-30 mm, $4-40 \mathrm{~mm}$.

\section{Experimental}

The investigated IR heaters integrated in the soldering station for mounting and dismantling of surface mounted components in various housings on a PCB. The IR station consists of systems of upper and lower heating, cooling, control unit and indicating device. The design provides for the variable use of two types of upper heating units with the 
possibility of their rapid replacement. Installation of heaters made so that their heating surface is located above the soldering zone. The lower heater, designed to preheat the circuit board to a temperature of $130-170^{\circ} \mathrm{C}$ in order to protect the mounted components and the board from thermal shock, includes two halogen lamps $1000 \mathrm{~W}$, a reflector and a heat dissipating plate. The cooling system includes three fans, two of which are located on the surface of the housing for cooling the soldering module and the upper heater and one that installed inside the housing to cool the lower heating block. The microcontroller control unit provides automated monitoring of the heating process and maintenance of the specified temperature profile, which in turn significantly improves the quality of soldering.

Temperature control is provided by means of a thermoelectric transducer (thermocouple type L), the signal from which through a direct current amplifier based on the operational amplifier is fed to the microcontroller. Using the built-in 10-bit ADC, the measured analog signal converted to a binary form. To minimize the measurement error, the amplified thermocouple signal measured 20 times per second with the subsequent calculation of the average value. The algorithm of calculations optimized for execution on microcontrollers of family MSP430. Taking into account the calculated temperature, the electromagnetic relays controlling the upper and lower heaters are controlled. The measured temperature, as well as the current status of the relay, are displayed on the liquid crystal display.

Temperature profiles for IR soldering of SMD components with a lower heating power of $1000 \mathrm{~W}$ are received using IR heaters in the near-IR region $(0.7-1.5 \mu \mathrm{m})$ - halogen IR lamp 300W and in the middle region $(2-10 \mu \mathrm{m})$ ceramic IR heater Elstein SHTS/4[6]. To automate the processing of data, the OVEN TRM210 measuring-regulator and a personal computer are used.

\section{Results and Discussion}

The thermal profiles of soldering halogen IR lamp KGM 30/300 and ceramic infrared heater Elstein SHTS/4 are shown in Fig. 3. At the stage of pre-heating, the shapes of the temperature profiles are close to each other. This is explained by the fact that at this stage the heating is carried out only by the bottom heater, which did not change during the experiment. For a halogen IR lamp, a 71-74\% higher heating rate is typical compared to ceramic heaters, which gives the basis for selecting this source as the main heating element in automated production lines with high output.

Analysis of the temperature profiles for soldering BGA with an IR heater Elstein SHTS/4 for distances of 10 and $30 \mathrm{~mm}$, showed that as the distance was increased three times the heating rate to the soldering temperature increased by 2-3 times. The shape of the isotherms of the heating fields of the halogen IR lamp indicates a high uneven soldering process where the maximum heating rate of $20-22{ }^{\circ} \mathrm{C} / \mathrm{s}$ is fixed on an area of up to 6-7 $\mathrm{mm}$ along the $\mathrm{X}$ axis and $4-5$ $\mathrm{mm}$ along the axis $\mathrm{Y}$, then through $3-4 \mathrm{~mm}$ towards the axes the heating rate decreases to $13-15^{\circ} \mathrm{C} / \mathrm{s}$, and then to 8-10 ${ }^{\circ} \mathrm{C} / \mathrm{s}$ after $4-5 \mathrm{~mm}$ (Fig. 4a).

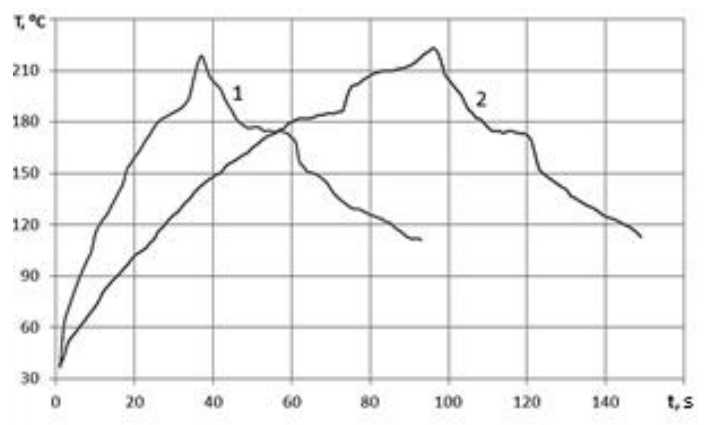

Fig. 3. Thermal profiles of soldering with: 1- halogen IR lamp, 2-ceramic IR heater.

Ceramic IR heater (Fig. 4b) has a relatively high uniformity of heating, the forms of thermal fields are symmetrical and do not depend on direction. The speed was $3-4^{\circ} \mathrm{C} / \mathrm{s}$ at a distance of $25 \mathrm{~mm}$ from the center, at $30 \mathrm{~mm}$ it decreases to 
$2-3^{\circ} \mathrm{C} / \mathrm{s}$ and at $35 \mathrm{~mm}-\mathrm{up}$ to $0.5-1{ }^{\circ} \mathrm{C} / \mathrm{s}$.

Based on the results of modeling the thermal fields for the heaters in the near and middle IR regions in the Solid Works 2012 (Flow Simulation) software package, the unevenness of the heating of the PCB and the housings of the installed components was obtained, which were: $34-36 \%$ and $26-44 \%$ for the near IR region, $4 \%$, and $8-12 \%$-for the average, respectively (Fig. 4). The best heating characteristics from the simulation results were obtained in the middle IR region, the near-IR region has the advantage only of the heating rate, so up to the soldering temperature $30 \mathrm{~mm}$ away from the board surface, the time was $30 \mathrm{~s}$ versus $60 \mathrm{~s}$ from the medium-wave radiator. The optimum distance from the surface of the module to the heating element should be within the range of $20-25 \mathrm{~mm}$, at which the heating irregularity is $3-5 \%$ for the components and $5-7 \%$ for the PCB.

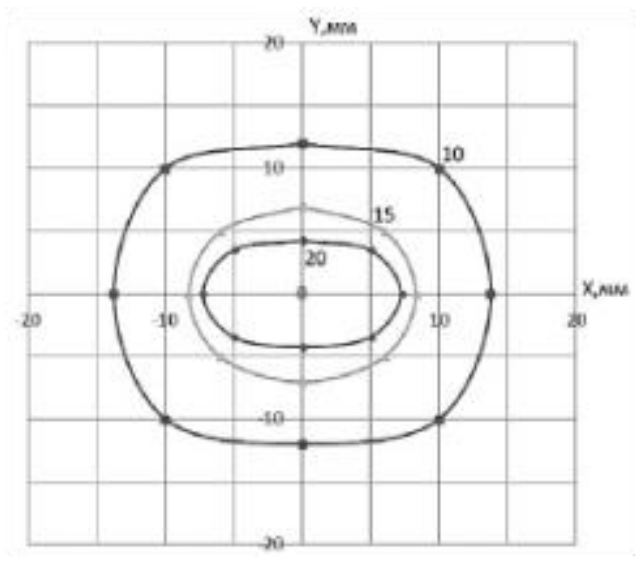

a

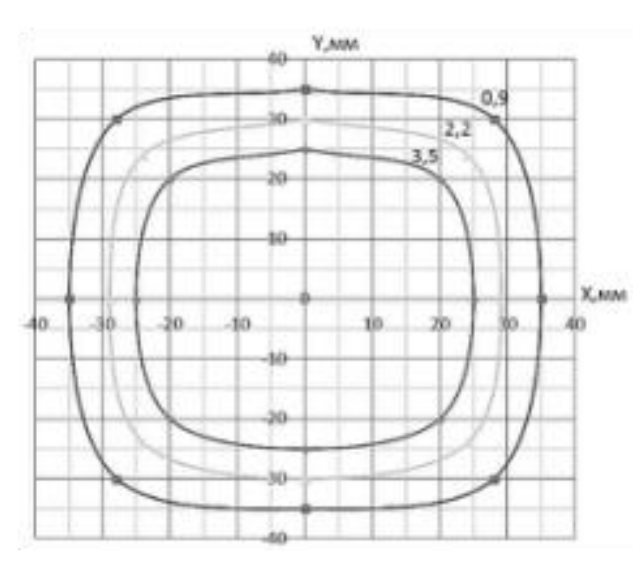

$\mathrm{b}$

Fig. 4. Thermal fields of the rate of IR heating, ${ }^{\circ} \mathrm{C} / \mathrm{sec}$ : a-halogen IR lamp $300 \mathrm{~W}, \mathrm{~b}$-Elstein SHTS / 4

Investigation of the temperature fields of the halogen incandescent lamp indicates a high process unevenness, when the maximum heating rate of $20-22^{\circ} \mathrm{C} / \mathrm{s}$ is reached at a distance of $4-7 \mathrm{~mm}$ from the center of the PCB. The ceramic IR heater showed an average heating rate of $3-4{ }^{\circ} \mathrm{C} / \mathrm{s}$ at a distance of $25 \mathrm{~mm}$ from the center, but the heating rate was decreased by a factor of 5-7 compared to a halogen IR lamp.

During the installation of surface-mounted components, contact joints were obtained. After examining the soldered connections of the installed components under the Carton NSWT-620. PFM-X microscope, it can be concluded that they comply with the IPC-A-610D standard.

\section{Conclusion}

Thermal field's models analysis shows that for a halogen infrared incandescent $300 \mathrm{~W}$ unevenness PCB heating was $45-55^{\circ} \mathrm{C}$ and in the enclosures of electronic components temperature unevenness- $90-100^{\circ} \mathrm{C}$. For ceramic IR heater Elstein SHTS $/ 4$ unevenness PCB heating is $8-13^{\circ} \mathrm{C}$, housings SMD components temperature differs from the board temperature: $\mathrm{BGA}-28-32^{\circ} \mathrm{C}, \mathrm{QFP}-24-26^{\circ} \mathrm{C}$ and $\mathrm{SMD}-5-20^{\circ} \mathrm{C}$.

Form experimental isotherm heating halogen lamp indicates unevenness of thermal fields where maximum heating rate $20^{\circ} \mathrm{C} / \mathrm{sec}$ is concentrated on an equal area $120 \mathrm{~mm} 2$. The ceramic IR heater has a higher uniformity of heating, but its application reduces the heating rate by a factor of 5-7 compared to the IR lamp, which was $3-4^{\circ} \mathrm{C} / \mathrm{s}$. Therefore, halogen IR lamps with a higher heating rate are the main heating element in automated lines of installation with high efficiency.

The use of ceramic IR sources average range optimally IR stations, intended for the repair of products with SMD 
components, since they require a high uniformity of the heating surface of the article during the installation work, and by increasing the heating time reduced thermal stresses in the volume of parts.

\section{References}

1. KLee NC. Reflow soldering processes and troubleshooting: SMT, BGA, CSP and flip chip technologies. Boston: Newnes, 2002, 20 (1).

2. Anguiano C, Felix V, Salazar D, Marquez H. Study of Heating Capacity of Focused IR Light Soldering Systems. Optics Express, 2013, 21(20): 23851-23855.

3. Norman RC. Near IR Reflow Soldering of Surface Mounted Devices. Surface Mounted Technology, 1986, 10: 27-30.

4. Felix M, Medel A, Bravo M, Marquez H, Salaza D. Heated area measurement and analysis of optical setups for focused IR light soldering system. IECON Proceedings (Industrial Electronics Conference), 2013, 3935-3939.

5. Lanin VL. Infrared Heating in the Technology of Soldering Components in Electronics. Surf. Eng Appl Electrochem, 2007, 43 (5): 381-386.

6. Lanin VL, Lappo AI, Lavor TE. Application Infra Red Heating for Mounting and Demounting SMD. Technology in Electronic Industry (Rus.), 2015, 3: 60-62. 